\title{
A Mobile Device Based ECG Analysis System
}

\author{
Qiang Fang, Fahim Sufi and Irena Cosic \\ School of Electrical and Computer Engineering, RMIT University \\ Australia
}

\section{Introduction}

Coronary heart disease (CHD) such as ischemic heart disease is the most common cause of sudden death in many countries including USA and Australia (Roberts, 2006). Its main manifestations consist of Acute Myocardial Infarction (AMI, or heart attack) and angina. Stroke (or cerebrovascular disease) is Australia's second biggest killer after CHD as well as the leading cause of long term disability in adults (Access Economics Pty Limited, 2005). Heart failure and stroke cause a big burden on society due to their high costs of care, lower quality of life and premature death. Among Australians having a heart attack, about $25 \%$ die within an hour of their first-ever symptoms and over $40 \%$ will be dead within a year (Access Economics Pty Limited, 2005). So, for the $40 \%$ who would generally be dead in one year, prognostic telemonitoring can be a life saver.

More than half of the 8000 home care agencies in US are currently using some forms of telemonitoring (Roberts, 2006). Only in US, sales of services and devices associated with telemonitoring are projected to rise from \$461 million in 2005 to over \$2.5 billion in 2010 (Roberts, 2006). Comparing with monitoring at hospital premises, home based telemonitoring not only provides great financial advantage but also gives patients freedom of staying home and living a normal life with their family. Moreover, rural hospitals with limited healthcare resources also benefit from telemonitoring service if those hospitals are connected with major advanced hospitals in metropolitan areas. The Electrocardiograph (ECG) is the electric signal generated by the heart activities. ECG is of significant diagnostic value to various cardiovascular and cerebrovascular diseases. ECG signal is one vital physiological signal that telemonitoirng systems normally pay attention.

A typical telemonitoring system includes medical signal or image acquisition, data storing, data analysis, and data transmission subsystems. Some advanced systems even incorporate data mining and knowledge management techniques. Thus a telemonitoring system needs to have sufficient data processing power and processing speed to handle the required high computation overhead.

With the recent advance in IC design, the computing power and the memory size of mobile device have increased considerably. This development makes many mobile devices, even some low end mobile phone handsets, capable of carrying out complex computing tasks. Especially after the recent release of iPhone and the powerful mobile programming platforms such as J2ME from Sun Microsystems ${ }^{\mathrm{TM}}$, Android from Google (code.google.com/android/), a mobile phone handset based telemonitoring solution has become possible (Roberts, 2006). 
In this chapter, we propose an electrocardiogram signal monitoring and analysis system utilizing the computation power of mobile devices. This system has good extensibility and can easily incorporate other physiological signals to suit various teleehalth scenarios. The system can be carried by both users, e.g., chronic patient, and the service providers, e.g., the medical doctors. Java ${ }^{\mathrm{TM}}$ based software running on the mobile phones performs computation intensive tasks like raw ECG data compression and decompression, encryption and detection of pathological patterns. The system can automatically alert medical service providers through Short Message Service (SMS) and Multimedia Message Service (MMS), when medical assistance is deemed crucial for the user based on the analysis results. Furthermore, in order to ensure the data interoperability and support further data mining and data semantics, a new XML schema is designed specifically for ECG data exchange and storage on mobile devices. This XML schema is named mECGML. The ECG data in mECGML format is tested in this application as a pilot study.

The rest of the paper is organized as follows. In the next section, the system architecture of the mobile phone based ECG analysis system is given. Section 3 presents a new XML schema specifically designed for ECG data storing and processing on mobile devices. In Section 4, the implementation of ECG R-R peak detection algorithms is described. Section 5 discusses the ECG signal visualization and transmission on mobile phone handset. The last section is a discussion on future work and conclusion.

\section{System architecture and programming environment}

\subsection{J2ME}

The core communication of the ECG monitoring system is done through the mobiles carried by both user and telemonitoring service provider. As our telemonitoring system targets on plain mobile phone handsets rather than iPhone or other high end handsets, the popular Nokia91 is chosen for this development. Most recent mobile phones support execution of miniature programs that utilize the mobile processing power. Java 2 Micro Edition (J2ME), .Net Compact Framework, Binary Runtime Environment for Wireless (BREW), Carbide $\mathrm{C} / \mathrm{C}++$ are some of the programming environments for mobile phone application development. J2ME is basically a subset of the Java platform designed to provide Java APIs for applications on tiny, small and resource-constrained devices such as cell phones, PDAs and set-top boxes. Among these languages J2ME is pervasively used, since the compact Java runtime environment, Kilobyte Virtual Machine (KVM), has been supported by a wide range of mobile phone handsets already. One major advantage of choosing Java is that a single program written in J2ME can be executed on a variety of mobile phones that support Java. Apart from the basic computation framework provided by KVM, each of the mobile phone also supports additional Java libraries for supporting additional functionalities such as Bluetooth connectivity, camera functionality and messaging services, etc. These additional libraries expose Application Programming Interfaces (APIs) to the programmer of the handset. J2ME architecture is composed of configuration and profile. Connected Limited Device Configuration (CLDC) defines the minimal functionalities required for a range of wireless mobile devices, e.g., mobile phone, PDA, Pocket PC, home appliances etc. Mobile Information Device Profile (MIDP) further focuses on a specific type of device like mobile phone or pager. MIDP also describes the minimum hardware or software requirement for a mobile phone. To the mobile application developer, both CLDC and MIDP expose Application Programming Interfaces (APIs) and functionalities supported by the KVM. 
Since the computational powers of the mobile phone handsets are expanding rapidly, current mobile phones possess considerable computation powers which can perform runtime complex tasks such as 3D games, MP3 and MPEG encoding and decoding. Even, Optical Character Recognition (OCR) software was tested on current mobile phones (Graham-Rowe, 2004). It is feasible to utilize the processors inside the mobile phone to process, compress, and transmit data in realtime for various telehealth applications. The realtime availability is of great importance for the sake of life saving. In principle, by careful design or selection of the proper computational algorithms, many complicated medical data processing and analysis tasks such as compression, decompression, encryption, correlation and transformation, feature extraction, and pattern recognition, can be implemented. However, the mobile phone platform supporting JavaTM language is subject to some software and hardware specific limitations. Unlike a Java runtime for PC, the KVM on mobile devices is a miniature version that can only run a subset Java APIs. Compared with a desktop PC, mobile phones based CLDC and MIDP restrict the usage of floating point operations, which means all the floating point must be removed before performing any operations on the mobile devices. Multi dimensional arrays are not supported as well; hence, any algorithm performing matrix based calculation needs to find an alternative approach. Luckily, those difficulties have been all successfully solved by sophisticated program skills employed in this project.

\subsection{System block diagram}

The architecture of our system is illustrated in Fig. 1. The mobile phone is the core part of this ECG telemonitoring system. The system is composed of total five subsystems, namely the patient's unit, the doctor's unit, the telephony network, the web based database system, and the server-side deployed intelligent analysis engine.

\subsection{Sub-systems}

\section{Patient's Unit}

This unit connects the biological acquisition device with the patient's mobile unit. Our own developed biosignal acquisition device with ECG, Oximetry, skin impedance and blood pressure sensors connects to the MIDlet software running on the patient's mobile via Bluetooth streaming. For the Bluetooth connectivity, JSR-82 (JSRS: Jave Service Request, 2008) specification was used. Third party Bluetooth enabled ECG monitoring device such as Alive Heart Monitor (www.alivetec.com) also can be used by this system.

Recently, a very new wireless interconnection technology, Near Field Communication (NFC) is being researched and on its way to be commercialized (NFC Forum web site, 2008). Study shows that the new generation NFC-Enabled phone will be widely available within next four years (Near Field Communications, 2008). Like Bluetooth, NFC based communication can easily be implemented to the proposed system, since the support for NFC with J2ME is already in progress through JSR-257 (JSRs: Java Service Requests, 2008). Third party sensors can also be supported for serving specialized purposes. Even sensors embedded with future generation mobile can be efficiently used through JSR-256 (JSRs: Java Service Requests, 2008).

Doctor's Unit

This unit is the specialized MIDlet software running on doctor's mobile handset. It serves the medical service provider's request of patient information as well as provides doctor with 


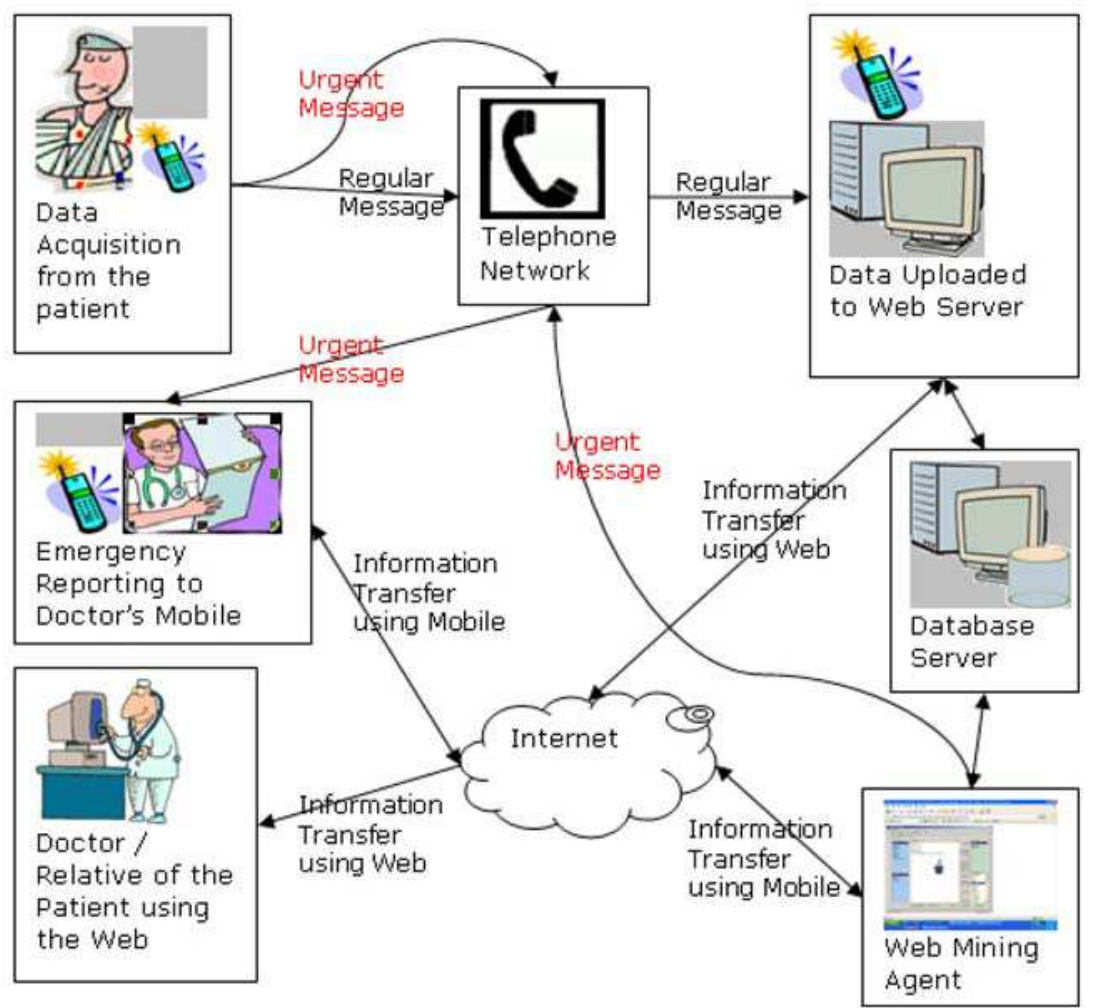

Fig. 1. System architecture of a mobile phone based ECG analysis system

the transmitted medical information and notifies the doctor in case of an emergency situation, where medical help is required the most. Custom reporting, charting and analysis of patient's condition for the selected period can be performed by the doctor's unit.

Web Server and Database

This unit provides restricted information to the authorized medical personnel or even relatives in case of elderly monitoring. All the biological signals from the patent's unit are stored here permanently. For implementation purposes we have chosen SQL Server 2000, a relational database, because of its support of data mining techniques (like, Clustering, Decision tree etc.) with Online Analytical Processing (OLAP) module. Intelligent Analysis Engine A web mining agent has been implemented. This agent is responsible for providing requested information to the doctor's mobile. Since our system is designed as a generic system it supports all the phones supporting Java ${ }^{\mathrm{TM}}$. Again, each phone (doctor's unit) is different in their display size, supported colors or even some operations. Web mining agent automatically analyses these information and presents formatted information to the doctor's mobile suitable for proper display. Moreover, web mining agent performs background data mining operations to analyze the stored information of patient. A feature database for various arrhythmia patterns has been created. The agent will perform a feature matching operation for any incoming ECG recording and make classification. If it discovers some pattern demanding urgent diagnosis, it can inform the doctor via SMS on a timely way. 


\section{Mobile ECG data exchange standard}

Extensible Markup Language (XML), a semi-structured data format, has been widely used for various data processing tasks such as text based data permanent storage, data exchange, and Web service. In order to enhance the data processing capability of conventional mobile devices for the sake of extensibility and interoperability, the adoption of XML is inevitable. Currently, there is no XML standard specifically designed for mobile devices from World Wide Web Consortium (W3C). The standard XML specification for ECG such as HL7's aECG and DICOM waveformat is too complicated and unnecessary to be fully implemented on mobile computing platforms. As a pilot study, we propose here a light-weighted XML Schema, named as mECGML, to facilitate the manipulation of ECG Data on mobile devices. There are a few XML parsers have been developed under J2ME environment (Knudsen, 2002). Among them, KXML 2.2 is chosen for its simplicity of use and compact size (only 9 $\mathrm{K})$.

\subsection{Existing ECG file formats}

There are many proprietary ECG data formats designed by different vendors for different applications. For example, PhysioNet uses a plain human readable text format containing signal, header, and annotation three components for physiological data including ECG. The PhysioNet format is not designed for machine operation. HL7 aECG is an annotated ECG format developed by HL7 Consortium. Its XML Schema is quite complicated. The ECG recording encoded in this format is a verbose file. SCP-ECG developed by European Union, is now approved as an international standard by ISO. SCP-ECG is not based on XML. It generates binary ECG files which are not human readable. Furthermore, DICOM, a standard for medical images, also proposed an auxiliary waveformat to integrate diagnostic ECG recordings. The DICOM waveforamt isn't based on XML either.

In many cases, the mobile devices are used for data transferring purpose rather than a data analysis platform. However, with the increasing power of the mobile devices, they can be further exploited to deal with complicated data processing tasks with sophisticated compact algorithms and proper programming skills. It is even possible to be used for data mining tasks. XML has been widely accepted as a standard for data exchange, storing and manipulation in a variety of application domains. Nevertheless, the use of XML in mobile devices is still limited due to aforementioned many computational constraints. Bearing this in mind, our newley designed light-weighted mECGML can be parsed by small or "tiny" XML parsers, such as KXML, in J2ME environment. The mECGML is designed to keep the essential information of the measured ECG data and has the capaibility to optionally keep pre-identified features for later intelligent data handling, such as data mining.

\subsection{The hierarchical representation of MECGML and the mECGML schema}

The design aim of mECGML is to provide an open standard for the storage, exchange and integration of ECG data among different mobile devices and other involved devices, e.g., acquisition devices, in an ambulatory monitoring applications. Normally, a remote or ambulatory monitoring system is an integrative system hence many incoming data are heterogeneous in nature. Table 1 shows the hierarchical representation of mECGML. 


\begin{tabular}{|c|c|c|c|c|}
\hline $\begin{array}{l}\text { Element/ } \\
\text { Attribute }\end{array}$ & Description & $\begin{array}{l}\text { Required/ } \\
\text { Optional }\end{array}$ & $\begin{array}{l}\text { Data } \\
\text { type }\end{array}$ & Example \\
\hline $\mathrm{mECG}$ & Root element & Required & Element & \\
\hline RecordTime & $\begin{array}{l}\text { The time when the } \\
\text { contained ECG } \\
\text { data was recorded; } \\
\text { the sampling rate } \\
\text { and the recording } \\
\text { duration are given } \\
\text { here as well }\end{array}$ & Required & Element & $\begin{array}{l}\text { <RecordTime> } \\
\text { <SamplingRate> } \\
350 \\
\text { </SamplingRate> } \\
\text { < StartingTime> } \\
\text { 2002-05-30T09:00:00 } \\
\text { </StartingTime> } \\
\text { <Duration> } \\
\text { PT160S } \\
\text { </Duration> } \\
\text { </RecordTime> }\end{array}$ \\
\hline RecordingDeviceDetail & $\begin{array}{l}\text { The description of } \\
\text { the recording } \\
\text { device }\end{array}$ & Required & Element & $\begin{array}{c}<\text { RecordingDeviceDetail } \\
\text { SerialNo="168312" > } \\
<\text { DeviceManufacturer } \\
\text { model="MAC501">GE } \\
</ \text { DeviceManufacturer }> \\
<\text { CalibrationTime> } \\
\text { 2008-06-30T10:00:00 } \\
<\text { CalibrationTime> } \\
<\text { Location }>10.7 .18\end{array}$ \\
\hline & & & & $\begin{array}{l}\quad</ \text { Location }> \\
</ \text { RecordingDeviceDetail }>\end{array}$ \\
\hline PatientDetail & $\begin{array}{l}\text { The description of } \\
\text { patient }\end{array}$ & Required & Element & $\begin{array}{l}<\text { PatientDetail } \\
\text { FirstName="John" } \\
\text { LastName="Smith" } \\
\text { PatientID="453212" /> }\end{array}$ \\
\hline RecorderDetail & $\begin{array}{l}\text { The description of } \\
\text { the acquisition unit } \\
\text { operator }\end{array}$ & Required & Element & $\begin{array}{l}<\text { RecorderDetail } \\
\text { FirstName="John" } \\
\text { LastName="Smith" } \\
\text { EmploymentID="44326" }>\end{array}$ \\
\hline EcgData & $\begin{array}{l}\text { The recorded raw } \\
\text { data; up to } 12 \text { leads } \\
\text { ECG data can be } \\
\text { stored }\end{array}$ & Required & Element & \\
\hline Feature & $\begin{array}{l}\text { The pre-identified } \\
\text { feature; the popular } \\
\text { features including } \\
\text { QRS complex, P } \\
\text { wave, ST segment, } \\
\text { T wave, etc. }\end{array}$ & Optional & Element & \\
\hline Annotation & $\begin{array}{l}\text { The annotation } \\
\text { made by } \\
\text { cardiologist }\end{array}$ & Optional & Element & \\
\hline
\end{tabular}

Table 1. Description of mECG element based on the hierarchical representation 
The detailed mECGML Schema (partial) is listed in Figure 2.

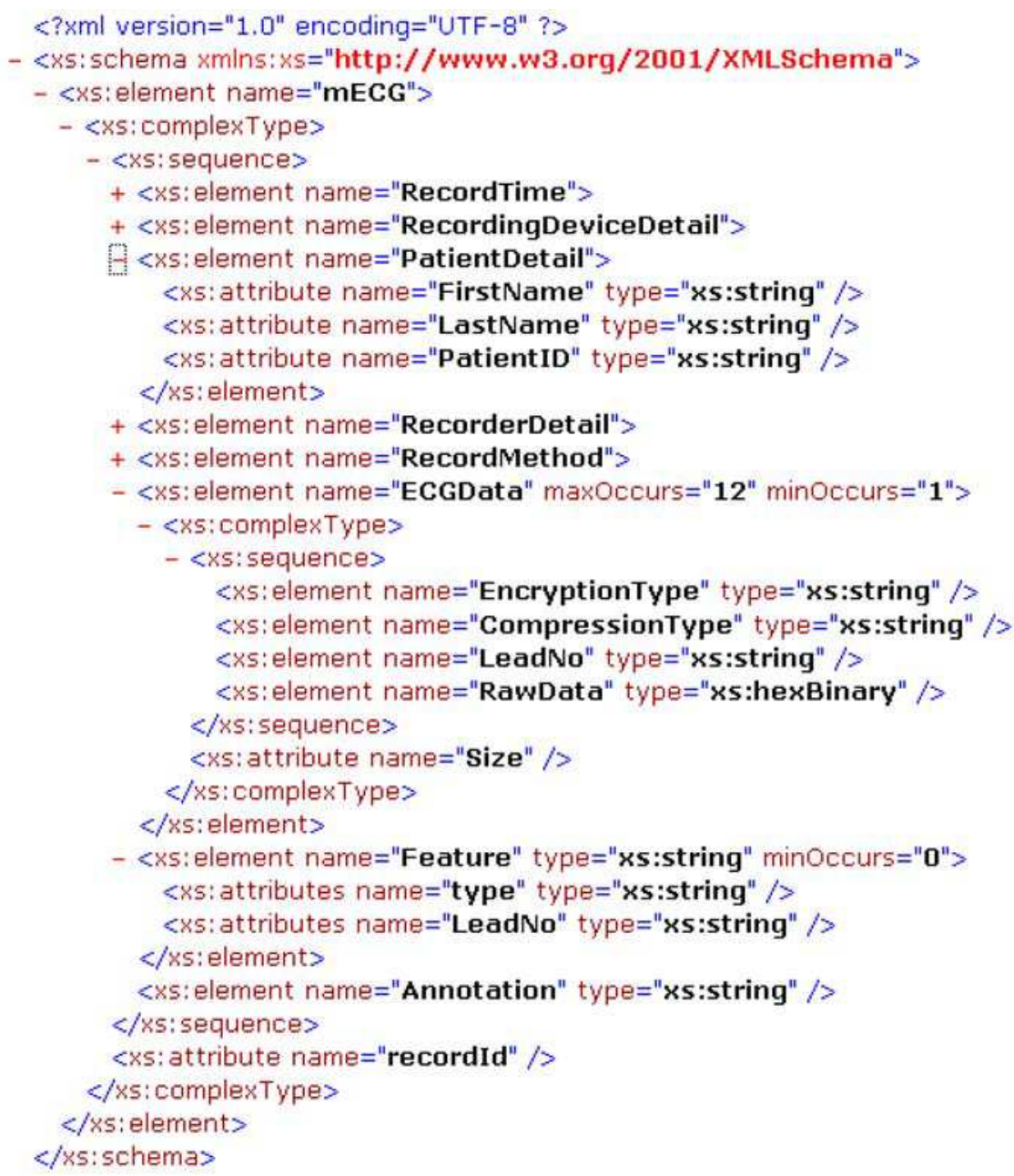

Fig. 2. The XML Schema of mECGML

\section{Wireless ECG transmission}

Mobile devices are predominantly used as a means of wireless transmission of signals (Jamemin et al, 2005). Earlier researchers used WAP gateways to transmit still images to the mobile phones (Zhao et al 2005). Since image files are generally larger than the text file files, WAP based technologies incur high usage of network bandwidth and slow transmission speed. We demonstrate here the possibility to transmit and receive biosignals in short text based messages and large binary files such as compressed ECG recordings via SMS and MMS using J2ME respectively. Graphical representation and image processing tasks can be performed by these J2ME miniature programs as well. 


\subsection{SMS and MMS}

SMS is predominantly used to send short text only messages containing a maximum of 160 characters. SMS supports two operations, Message Originating (MO) and Message Terminated (MT). MO is for sending SMS and MT is for receiving SMS. Once an SMS is sent from a mobile phone, the message arrives at Short Message Service Center (SMSC). SMSC generally follows Store \& Forward rule, which means message is stored inside SMSC until it reaches the recipient. Hence, an SMSC constantly tries to transmit the SMS until it is received by the recipient. Some SMSCs are guided by Forward \& Forget rule which means after sending the SMS, the SMSC deletes the message from the server. Therefore receipt of the SMS is never guaranteed. Both text and binary data of limited length can be transmitted by SMS.

MMS utilizes the concept of other messaging services like SMS, Mobile Instant Messaging and Mobile E-Mail. Once a sender sends an MMS message, the receiver is notified via an SMS, followed by establishment of a WAP connection to download the MMS message. Unlike SMS, MMS provides support for transmission of text, audio, video and image files. A typical MMS file has a MMS header and MMS body. MMS body contains one or more Mulitpart Messages. Each of those Multipart Messages is composed of their own header and body. MMS supports emailing the message to multiple recipients (to, $\mathrm{cc}$, bcc). The crucial benefit of MMS over SMS is the message length. But some of the telecommunication service providers impose a limitation in file size to reduce their network congestion. Mobile handsets also have regulated file size restrictions. It is possible to transmit larger files through MMS with minimal restrictions, e.g., each of the message part containing texts should be less than $30 \mathrm{~Kb}$.

\subsection{System design and implementation}

To serve our purpose of automated sending and receiving of biosignals through SMS and MMS, we used JSR-205 library, which was included with our Mobile Phone Platform (Nokia N91). JSR-205 (Wireless Messaging API 2.0) is the update of JSR-120 (Wireless Messaging API 1.1). JSR-120 only supports CBS/SMS with text or binary input. But JSR-205 enhanced its support to Multipart MMS Messages. For drawing curves and graphic display on Mobile handset screen, the J2ME Graphic Library was utilised.

In a practical remote monitoring scenario, the patient is connected with an ECG monitor as well as devices for other physiological signals such as blood pressure, pulse and SpO2. The acquisition system transmits data to the patient's mobile phone via Bluetooth Connectivity. Different types of third party physiological acquisition systems with different data output formats can be used. The Mobile phone parses those input data in proprietary format and wraps the ECG data together with metadata into mECGML format. The generated mECGXML file is further compressed to reduce the transmission overhead. Then the mobile handset sends SMS and MMS packets, which will be forwarded to the SMS Center (SMSC) and MMS Center (MMSC). From SMSC and MMSC the biosignals (Blood pressure, Pulse \& ECG) will disembark at the doctor's mobile phone. As soon as the SMS/MMS packets reach the doctor's mobile phone, the Java software installed in the doctor's mobile phone decompresses and parses the ECG recording, followed by the creation of graphs, curves and other signal processing tasks. Fig. 3 illustrates the whole telemonitoring scenario. For our experiments we developed a pair of J2ME based Java software (Called MIDlet), which were running in both patient's \& doctor's mobile phone. 


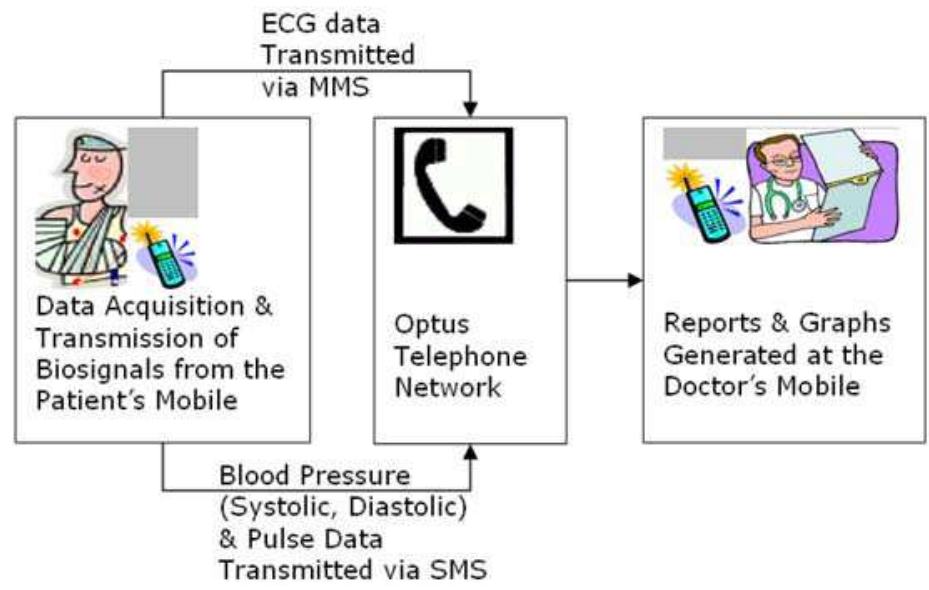

Fig. 3. Biosignal transmission via SMS and MMS

\subsection{Patient's mobile phone}

The MIDlet for patient's mobile phone is responsible for sending blood pressure and pulse data via SMS and compressed ECG data via MMS. SMS packets were created using the format presented in Fig. 4. as comma separated values. Because of the SMS length restriction only 15 hourly data could be accommodated using this format. For each hour Systolic Blood Pressure (SBP), Diastolic Blood Pressure (DBP) and Pulse (P) were sent via SMS. In the trial experiment, Siemens C75 hand set was utilised as the patients' handset to send SMS to the Doctors Mobile phone.

\begin{tabular}{l} 
SMS Packet Format \\
a. $\mathrm{H}_{1}, \mathrm{H}_{2}, \mathrm{H}_{3}, \mathrm{H}_{4}, \mathrm{H}_{5}, \mathrm{H}_{6}, \ldots, \mathrm{H}_{15}$ \\
b. $\mathrm{H}_{\mathrm{n}}=\mathrm{SBP}_{\mathrm{n}}, \mathrm{DBP}_{\mathrm{n}}, \mathrm{P}_{\mathrm{n}}$ \\
c. Length (SMS Message a.) $<=160$ \\
\begin{tabular}{|l} 
SMS Example \\
"65,120,78,70,125,80,..,80,130,82" \\
MMS Example: \\
-0.170 \\
-0.155 \\
-0.175 \\
-0.180 \\
-0.190 \\
-0.180 \\
$\ldots$
\end{tabular} \\
\hline
\end{tabular}

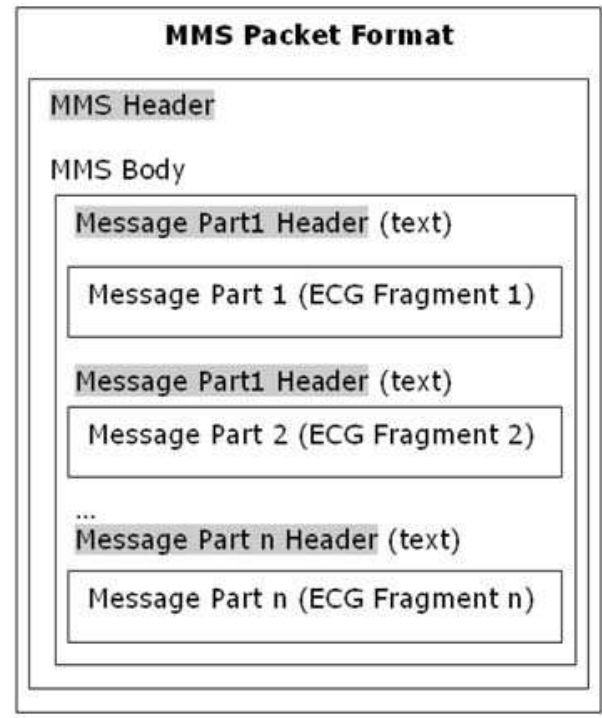

Fig. 4. SMS and MMS Packets $(\mathrm{H}=$ Hourly Data, $\mathrm{SBP}=$ Systolic Blood Pressure, $\mathrm{DBP}=$ Diastolic Blood Pressure, $\mathrm{P}=$ Pulse ) 
Meanwhile, for ECG data via MMS, it is possible to send files in bigger size, since our mobile phone supports up to $330 \mathrm{~Kb}$ of MMS data and the telephony network (Optus, Australia) doesn't impose any file size restriction. However, from our development platform (NetBeans IDE 5.5) a size restriction of 30k was imposed for each of the Message Part (for text data). Hence, to accommodate 1 minute data, which was about $157 \mathrm{~K}$, we created 6 Message Parts by the segmentation of the ECG file. Following this procedure each of the 6 ECG segments became 10 second long ECG message part (text). Nokia N91 handset was used to send MMS message, since it supports JSR 205. Siemens C75 couldn't be exploited for sending MMS because of its lack of support for JSR 205 (it only supports JSR120).

\subsection{Doctor's mobile phone}

In the doctor's mobile phone, listeners were installed for listening to both SMS and MMS messages. To distinguish between messages sent from the patient and normal messages (sent from family, friends or colleagues) specific ports (for SMS) and Application ID (for MMS) was exploited. Hence, the patient's handset needs to send SMS and MMS messages to those predefined ports and application IDs. If messages without specifying any ports are sent from the patient's mobile, the server connection of the doctor's mobile phone would not be able to process those messages, instead, the mobile's own message handler (Inbox) will receive those messages. Port 5000 was used for sending and receiving of SMSs and Application ID com.ecg.mms was used for sending and receiving MMSs.

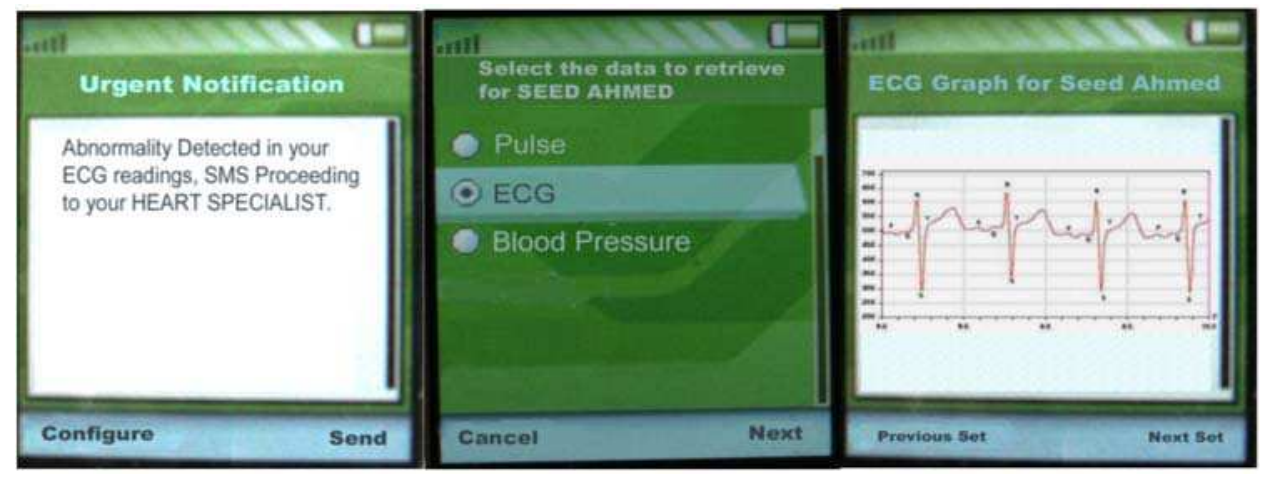

Fig. 5. Screen shot of the User Interface of Doctor's mobile

Once the messages containing biosignals are received and identified by the doctors mobile phone, Canvas class of J2ME is instanced and graphics are drawn on the doctor's mobile phone in realtime. Before the actual drawing of the curves and graphs, the text based SMS and MMS messages are transformed to multiple integer values. From those integer values appropriate coordinate system is generated, since mobile phone's origin of coordinate is located at the upper left corner of the screen. Therefore, generating curves and graphs of biosignals needs proper calculation and translation before drawing then on to the mobile phone's screen. Fig. 5. is the screenshot taken from a doctor's handset which shows the end result of menu and graphing.

\subsection{Results}

We successfully demonstrated that biosignal transmission via SMS and MMS using J2ME is a realistic solution. Mobile software can be programmed to automate the process of 
biosignal collection, noise identification, noise reduction, message packet creation and message transmission. On the other hand, realtime generation of curves and visual displays provide assistance for prompt medical decision. During our experimentation, we generated 12 SMSs and 9 MMSs. SMS Messages sent by the sender were always received by the receiver within one minute (12 out of 12 times). However, for only 2 cases of MMS messages, more than 1 minute time was consumed by the network operator. This result implies the fact that for monitoring less serious patient's, SMS and MMS transmission can be adopted, since these messaging techniques do not assure timely and dependable delivery.

\section{ECG R-R peak detection}

\subsection{R-R peak and HVR}

Electrocardiogram or ECG is the record containing electrical activities of the heart. ECG is widely used to diagnose different heart abnormalities. Different patterns of a normal ECG graph are denoted by $\mathrm{P}, \mathrm{Q}, \mathrm{R}, \mathrm{S}$ and T. Detection of ECG RR interval and QRS complex from the recorded ECG signal is crucial for a sustainable health monitoring scenario, since a wide range of heart diseases like tachycardia, bradycardia, arrhythmia, palpitations etc. can be efficiently diagnosed utilizing the resultant RR interval. Many different RR interval calculation algorithms have been proposed. We selected a few preferred algorithms, which were previously executed only on PC environment for realtime RR detection, and deployed them in mobile phones to ascertain their suitability and performances. The experiment results suggest that mobile phones do possess the computation power to detect the RR peak in realtime. By knowing the RR peak, it is easy to further calculate the Heart Rate Variability (HRV). HRV is the rhythmic alteration in heart rate. Although there have been many researches conducted on HRV, the understanding of HRV is still not completed. It is generally accepted that HRV is an important index for heart condition in two aspects: the high frequency part $(0.18-0.4 \mathrm{~Hz})$ is correspondent to respiration and the low frequency part (0.04-0.15 Hz) is related to vagus and cardiac sympathetic nerves. The fact that HRV is of great prediction value to heart attack survivors has been evidenced by a reported correlation between reduced HRV and the patient death []. There are many different approaches to assess the HRV. The tachogram is used in this study.

Fig. 6 shows an abnormal ECG recording representing the first 899 samples ( 2.5 seconds) of entry 232 of MIT-BIH arrhythmia database (MIT-BIH Arrhythmia Database, 2007). 12 ECG entries each with 60 seconds measurement from MIT-BIH arrhythmia database were used for our experimentation. MIT-BIH database has been extensively used in literature for performance monitoring and comparison of different algorithms that perform ECG signal processing (Friesen, 1990). In this research three fundamental QRS and RR interval detection algorithms namely Amplitude Based, First Derivative Based and Secondary Derivative Based techniques, have been chosen as a pilot study to implement ECG R-R peak detection on mobile phone.

\subsection{Amplitude Based Technique (ABT)}

The Amplitude based technique (ABT) performs very simple comparison where the ranges of sample ECG points falling beyond an amplitude threshold are determined to be a QRS complex candidate. For Fig. 6, the amplitude threshold can be 0.2. After the QRS complex is 
detected, the highest amplitude of the detected QRS is ascertained to be R peak. Equation (1)-(4) generalizes the amplitude based method. The original ECG signal, $x_{n}$, from the patient body is given by (1).

$$
\mathbf{x}_{n}=x_{1}, x_{2}, \ldots, x_{N}
$$

where, $n=1,2, \ldots, N$ and $N$ is the length of the signal.

$$
\left(x_{r}, x_{r+1}, x_{r+2}, \ldots, x_{r+k}\right), \ldots,\left(x_{l}, x_{l+1}, x_{l+2}, \ldots, x_{N-c}\right)>\text { amplitude threshold }
$$

where, $1<r<1<\mathrm{N}, \mathrm{x}_{\mathrm{N}-\mathrm{c}}$ is the last value greater than the threshold and both $\mathrm{x}+\mathrm{k}+1$ and $\mathrm{x} \mathrm{nc}+$ 1 are less than the amplitude threshold.

Each of the section enclosed by the parenthesis of (2) (left side of the equation) is QRS complex candidate.

$$
\begin{gathered}
R \text { peak }=\operatorname{Max}(Q R S \text { Complex }) \\
R R \text { Interval }=\frac{n_{r}}{f}
\end{gathered}
$$

where, $n_{r}$ is the number of samples between two corresponding $\mathrm{R}$ peaks and $f$ is the sampling frequency of the ECG.

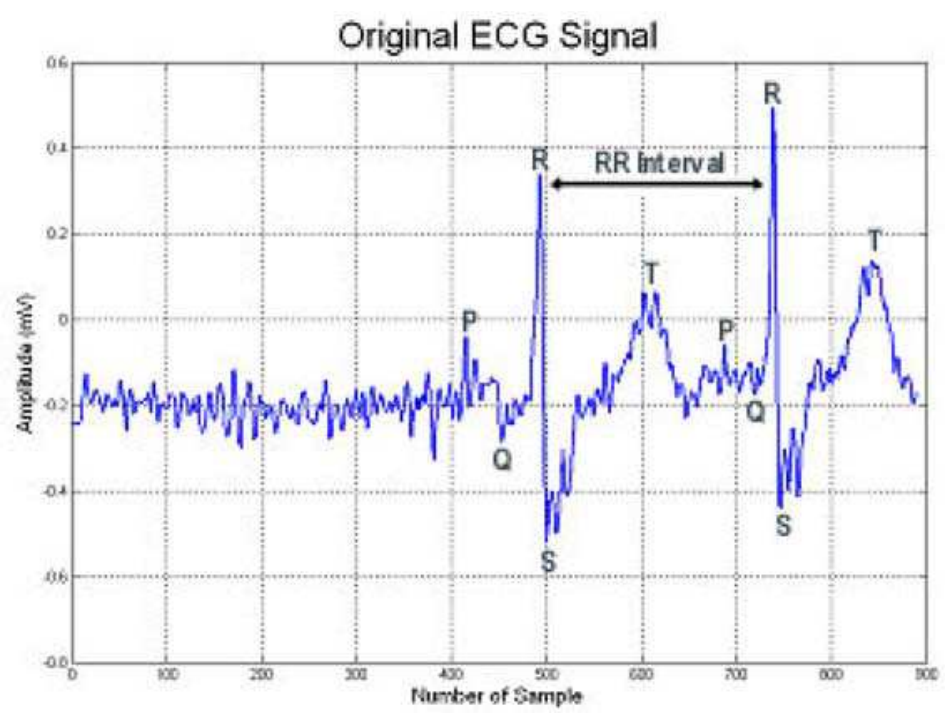

Fig. 6. An original abnormal ECG sginal

\subsection{First Derivative Based Technique (FDBT)}

This method is also called Hamilton-Tompkins method. The QRS detection is achieved by performing first derivatives of the ECG samples. This is because the QRS complex generally 
has the steepest slope and has the maximal magnitude as well. RR interval can be measured by several first derivatives based QRS detection techniques described in the literature (Mahoudeaux, 1981; Menrad et al., 1981; Hosinger et al, 1971) followed by the application of (3)-(4).

To measure the performance of FDBTs in mobile phone platform, slightly modified version of [4] was adopted. The first derivative, $\mathrm{y} n$ was calculated at each sample point of $x_{n}$ such that

$$
y_{d}=x_{n+1}-x_{n-1}
$$

where, $d=1,3, \ldots, N-1$

A QRS complex is detected whenever three consecutive first derivative values are greater than a positive slope threshold, followed within next ten samples by two consecutive first derivative values less than a negative slope threshold. Equation (6)-(8) describes the process

$$
\begin{gathered}
y_{i}, y_{i+1}, y_{i+2}>0.1375 \\
y_{j}, y_{j+1}<-0.2 \\
j-\mathrm{i}<10
\end{gathered}
$$

After the detection of the QRS complex Eq. (3-4) is used to derive the RR interval.

\subsection{Second Derivative Based Technique (SDBT)}

Previous research reveals several second derivative based QRS detection algorithms [8, 9]. For performance comparison, a modified version of [8] was used. At first, Eq. (9-10) was used to evaluate the absolute values of first $\left(y^{0}\right)$ and second $\left(y^{1}\right)$ derivative.

$$
\begin{gathered}
y 0_{d}=A B S\left(x_{n+1}-x_{n-1}\right) \\
y 1_{s}=A B S\left(y 0_{n+2}-2 * y 0_{n}+y 0_{n-2}\right)
\end{gathered}
$$

where, $s=1,2,3 \ldots, N-3$

A scaling value, $y 2$ is obtained from Eq. (11).

$$
y 1_{s}=A B S\left(y 0_{n+2}-2 * y 0_{n}+y 0_{n-2}\right)
$$

For all values exceeding a threshold, are determined to be the start of a QRS candidate (Eq. (12)).

$$
y 2_{d} \geqslant 0.9
$$


Values from $x_{d-3}$ to $x_{d+3}$ is passed to Eq. (3) as the QRS Complex to compute R peak and finally Eq. (4) is used to calculate the RR interval. After locating a single R peak the next seven $y 2_{d}$ values are ignored, since most of time there are few $y 2_{d}$ values greater than the threshold surrounding a single R peak.

\subsection{Algorithm implementation and results}

Since, plain mobile phones are constrained with various hardware and software limitations and capable of executing only 3000 to 10000 operations per second, selection of RR detection algorithms was focused mainly on lower complexity level in this study. Three different RR detection algorithms representing three pioneering classifications namely amplitude based, first derivative based and second derivative based RR detection algorithms, were selected for running inside the mobile phones. The RR detection algorithms were then programmed for Java based mobile phones. Sun Java Wireless Toolkit 2.5 was used for programming MIDlets in regular mobile phones. Performances were compared for three different mobile phones (Nokia 91, Nokia 6280 and Siemens C75) using 12 randomly selected ECG entries from MIT-BIH Arrhythmia database. These three models are all popular and regular mobile phones within middle price range.

The ECG entries of MIT-BIH were kept inside the mobile phones during the testing and performance monitoring of the RR detection algorithms. Therefore, during that period Bluetooth was not used for sending ECG to the mobile phone from the acquisition device.

The ECG signal has single channel, 11 bit resolution with $360 \mathrm{~Hz}$ sampling frequency and yields a step size of $5 \mathrm{RV}$. They contained 60 seconds ECG signal meaning each of the selected MIT-BIH entries had total $21600(360 * 60)$ samples.

Table 2 demonstrates the processing time requirement for different RR detection algorithms using different randomly selected ECG files on three different mobile phones. A realtime factor, Rf was calculated using (13).

\begin{tabular}{|l|l|l|l|l|l|l|l|l|l|}
\hline \multirow{2}{*}{$\begin{array}{l}\text { MIT } \\
\text { BIH } \\
\text { Entry }\end{array}$} & \multicolumn{3}{|l|}{ Nokia N91 } & \multicolumn{2}{l|}{ Siemens C75 } & \multicolumn{2}{l|}{ Nokia 6280 } \\
\cline { 2 - 10 } & $\begin{array}{l}\text { ABT } \\
(\mathrm{ms})\end{array}$ & $\begin{array}{l}\text { FDBT } \\
(\mathrm{ms})\end{array}$ & $\begin{array}{l}\text { SDBT } \\
(\mathrm{ms})\end{array}$ & $\begin{array}{l}\text { ABT } \\
(\mathrm{ms})\end{array}$ & $\begin{array}{l}\text { FDBT } \\
(\mathrm{ms})\end{array}$ & $\begin{array}{l}\text { SDBT } \\
(\mathrm{ms})\end{array}$ & $\begin{array}{l}\text { ABT } \\
(\mathrm{ms})\end{array}$ & $\begin{array}{l}\text { FDBT } \\
(\mathrm{ms})\end{array}$ & $\begin{array}{l}\text { SDBT } \\
(\mathrm{ms})\end{array}$ \\
\hline 100 & 6 & 8 & 8 & 5 & 6 & 6 & 1 & 1 & 2 \\
\hline 102 & 6 & 7 & 7 & 5 & 6 & 6 & 1 & 1 & 1 \\
\hline 105 & 6 & 6 & 8 & 5 & 5 & 6 & 1 & 1 & 1 \\
\hline 114 & 6 & 7 & 7 & 5 & 6 & 6 & 1 & 1 & 1 \\
\hline 117 & 7 & 8 & 7 & 6 & 6 & 6 & 1 & 1 & 1 \\
\hline 201 & 6 & 7 & 7 & 4 & 5 & 5 & 1 & 1 & 1 \\
\hline 213 & 7 & 7 & 8 & 4 & 5 & 6 & 1 & 1 & 1 \\
\hline 219 & 6 & 7 & 7 & 4 & 6 & 6 & 1 & 1 & 1 \\
\hline 222 & 6 & 7 & 7 & 5 & 5 & 6 & 1 & 1 & 1 \\
\hline 228 & 7 & 7 & 8 & 6 & 6 & 6 & 1 & 1 & 1 \\
\hline 231 & 6 & 7 & 7 & 5 & 6 & 6 & 1 & 1 & 1 \\
\hline 234 & 7 & 7 & 7 & 5 & 5 & 6 & 1 & 1 & 1 \\
\hline
\end{tabular}

Table 2. Performance Comparison of The RR Interval Algorithms Among 3 different Mobile Phone Handsets. 


$$
R_{f}=\frac{P_{t}}{\text { Measurement window }}
$$

where, $\mathrm{Pt}$ is the processing time needed to run the RR detection algorithm for an individual ECG entry within one measurement window and the window is 60 seconds in this study. Pt is measured by time stamps embedded in the beginning and the ending of the implementation codes. Whenever (14) is true, the algorithm is classified as realtime for mobile phone based processing.

$$
R_{f}\langle<1
$$

Equation (14) basically exemplifies the simple fact that to operate the algorithm in realtime, the processing time required to process 1 seconds ECG data must be much less than 1 second.

By using Eq. 14, the realtime factor can be calculated for all three RR detection algorithms implemented on three different mobile devices. It is shown that the value of Rf ranged from $1.6 \times 10-5$ to $13.3 \times 10-5$ which are much less than 1 during the entire experimentation process. Therefore, for all the mobile phones tested, realtime operations were achieved based on the criteria set in this study and Nokia 6280 was found to consume the least processing time. Though the data acquisition time isn't taken into account in this detection program, it will unlikely affect the experiment result due to multi-thread supporting of J2ME. The research of realtime RR peak detection on mobile phone is the foundation step for the future research of mobile phone based abnormality monitoring and identification from realtime acquired ECG recordings. Mobile phones based RR detection is certainly a cheaper solution since expensive PCs are not required for data processing tasks anymore. In addition, a mobile phone based system provides a true ambulatory and wireless connectivity for the patient and other needed user groups. The monitoring and pre-analysis results can be easily transmitted to remote locations using 3G, GPRS, SMS, CBS, MMS, Email, HTTP etc. or even it can be simply displayed on the mobile phone screen.

\section{Conclusion and future work}

We propose in this chapter a mobile phone based intelligent ECG telemonitoring system with good extensibility. The vital ECG signal can be acquired, analyzed locally, transmitted, and analyzed remotely in a quasi-realtime sense. The computation power of mobile handset is extensively harnessed to identify major ECG morphological abnormalities and activate the early warning mechanism via both SMS and MMS. We also designed a light weighted ECG data format, mECGML, to facilitate the seamless data integration within the system. The ECG data is also stored natively in this format in the server side.

The proposed system makes it easy to change the functionalities or telemonitoring applications, just by updating the program by Over-the-Air (OTA) deployment (Yuan 2004) of MIDlets to the mobile. So, the system can be adopted for different application with minimal effort. Most of all, J2ME based system reveals standard and different third party APIs for performing complex computations like, compression, encryption, steaming 
multimedia for the service of telehealth within the mobile phone. The proposed platform provides flexibility in terms of wireless communication, from the ECG signal acquisition device to mobile through Bluetooth and from the patient to service provider communication via $2.5 \mathrm{G}$ or $3 \mathrm{G}$ network.

The proposed system enables the doctor to receive/analyse the patient report and also, deliver doctor's treatment and specialist advice to remote patient. The doctor doesn't need to be sitting in front of a stationary computer within the medical facility. The proposed system uses smart client technology instead of thin client technology used by some WAP based system (Hung \& Zhang, 2003). So, it can use the network bandwidth much more efficiently. Moreover, it doesn't need any physical wired internet connection like some systems (Chen 2004; Jin-gang et al, 2005; Braecklein et al, 2005; Hung \& Zhang, 2003; Shieh et al, 2005; Glaros et al, 2003), making the proposed system a true wireless solution. In addition, it doesn't have any restricted coverage area. It is accessible within the global mobile coverage area. The sending of physical parameters from the acquisition device is automated by the MIDlet running in patient's mobile, making the whole system less error prone compared to some existing system (Zhou et al, 2005). Finally the proposed system is based on generic mobile phone (costing 100s of dollars) supporting Java KVM, instead of expensive Pocket PC, Smart phone or iPhone (1000s of dollars) based solution. According to some recent studies (Baker 2006), cost of home monitoring unit is needed to be drop below 1000 USD to overcome the limited usage of telemonitoring devices. In this respect, cost effectiveness is another crucial advantage that the proposed system provides.

During our experimentation we used both SMS and MMS for transmission of medical information in both text format and binary format. At this stage, only compression ECG data is wrapped into MMS format, in the future, we will try on the transmission of different medical images files such as CT, MRI, X-ray and ultrasound images, using MMS on mobile. The implementation of DICOM standard will also be test on mobile device.

As a summary, the presented ECG telemonitoring system is a cost effective, flexible and robust solution supporting a unique mobile based computational platform where compression, detection and even encryption are some of the possibilities.

\section{References}

Access Economics Pty Limited (2005) The shifting burden of cardiovascular disease in Australia, A report of Heart foundation. [Online]. Available: www.heartfoundation.com.au/media/nhfa_shifting_burden_cvd_0505.pdf

Baker, M.L. (2006) Study: Medicare, Insurers Reluctant to Pay for Telehealth. [Online]. Available:

http://www.eprescribingnews.com/archives/2006/04/study_medicare.html

Braecklein, M. et al (2005). Wireless Telecardiological Monitoring System for the Homecare Area, Proceedings of the 27th Annual Conference of IEEE Engineering in Medicine and Biology, September 2005

Chen, Y. Y. et al (2004). Development of wireless blood glucose meter and diabetes selfmanagement system, Proceedings of the 26th Annual International Conference of the IEEE EMBS, September 2004, pp. 3384-3386 
Friesen, G. et al (1990). A Comparison of the Noise Sensitivity of Nine QRS Detection Algorithms. IEEE Transactions on Biomedical Engineering, Vol. 37, No. 1, pp. 8598

Glaros, C. et al (2003). A wearable intelligent system for monitoring health condition and rehabilitation of running athletes, Proceedings of 4th IEEE Conf. on Information Technology Applications in Biomedicine, pp. 276-279, 2003

Graham-Rowe, D. (2004). Camera phones will be high-precision scanners, NewScientist.com news $\quad$ service. [Online]. Available:

http:/ / www.newscientist.com/article.ns?id=dn7998

Hosinger, W.P. et al.(1971). A QRS pre-processor based on digital differentiation. IEEE Trans. Biomed. Eng. Vol. 8, pp. 212-217, 1971

Hung, K. and Zhang, Y. T. (2003). Implementation of a WAP-based telemedicine system for patient monitoring. IEEE Transactions on Information Technology in Biomedicine, Vol. 7, No. 2, pp. 101- 107

Knudsen, J. (2002). Parsing XML in J2ME. [Online]. Available: http://developers.sun.com/mobility/midp/articles/parsingxml/

Jin-gang, W. et al (2005). Remote Heart Sound Monitoring System, Proceedings of the 27th Annual Conference of IEEE Engineering in Medicine and Biology, September 2005

JSRs: Java Service Requests (2008). Java Community Press Web Site. [Online]. Available: http://www.jcp.org/en/jsr/overview

Mahoudeaux, M. (1981). Simple microprocessor based system for online ECG analysis, Med. Biol.Eng. Comput., Vol 19, pp. 497-500

Menrad, A. et al. (1981). Dual microprocessor system for cardiovascular data acquisition, processing and recording, Proc, 1981 IEEE Int. Conf. Industrial Elect. Contr. Instrument, pp. 64-69

MIT-BIH Arrhythmia Database (2007). [Online]. Available: http://www.physionet.org/cgibin/ rdsamp

Near Field Communications (NFC) (2008). Simplifying and Expanding Contactless Commerce, Connectivity, and Content. ABI Research, Oyster Bay, NY, 4Q2005

NFC Forum web site (2008). [Online]. Available: http:/ / www.nfc-forum.org/

Roberts, R. (2006). Use of Remote Monitoring Devices Increases, Telemedicine Information Exchange, (Original Source: Wall Street Journal, April 18, 2006). [Online]. Available: http://tie.telemed.org/legal/news.asp

Shieh, J. S. et al (2005). Web-Based Remote Monitoring Health of the elderly via mobility changes using frequency and rank order statistics, Proceeding of the IASTED International Conference of Telehealth, July 2005

Yuan, M. J. (2004). Enterprise J2ME: developing mobile Java, Upper Saddle River, NJ: Prentice Hall PTR, c2004

Zhao, E. and Cui, L. (2005). EasiMed: A remote health care solution, Proceedings of the 27th Annual Conference of IEEE Engineering in Medicine and Biology, September 2005 
Zhou, H. et al (2005). A Real-Time Continuous Cardiac Arrhythmias Detection System: RECAD, Proceedings of the 27th Annual Conference of IEEE Engineering in Medicine and Biology, September 2005 


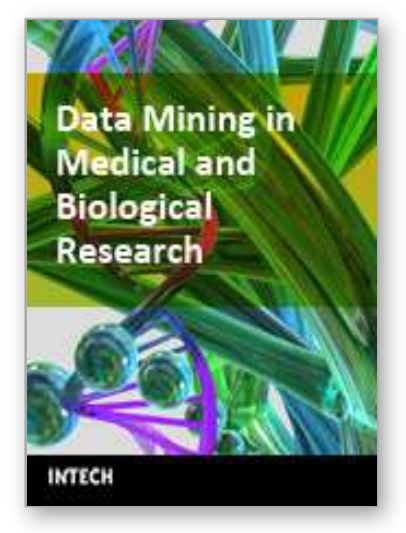

\section{Data Mining in Medical and Biological Research \\ Edited by Eugenia G. Giannopoulou}

ISBN 978-953-7619-30-5

Hard cover, 320 pages

Publisher InTech

Published online 01, November, 2008

Published in print edition November, 2008

This book intends to bring together the most recent advances and applications of data mining research in the promising areas of medicine and biology from around the world. It consists of seventeen chapters, twelve related to medical research and five focused on the biological domain, which describe interesting applications, motivating progress and worthwhile results. We hope that the readers will benefit from this book and consider it as an excellent way to keep pace with the vast and diverse advances of new research efforts.

\section{How to reference}

In order to correctly reference this scholarly work, feel free to copy and paste the following:

Qiang Fang, Fahim Sufi and Irena Cosic (2008). A Mobile Device Based ECG Analysis System, Data Mining in Medical and Biological Research, Eugenia G. Giannopoulou (Ed.), ISBN: 978-953-7619-30-5, InTech,

Available from:

http://www.intechopen.com/books/data_mining_in_medical_and_biological_research/a_mobile_device_based_ ecg_analysis_system

\section{INTECH}

open science | open minds

\section{InTech Europe}

University Campus STeP Ri

Slavka Krautzeka 83/A

51000 Rijeka, Croatia

Phone: +385 (51) 770447

Fax: +385 (51) 686166

www.intechopen.com

\section{InTech China}

Unit 405, Office Block, Hotel Equatorial Shanghai

No.65, Yan An Road (West), Shanghai, 200040, China

中国上海市延安西路65号上海国际贵都大饭店办公楼405单元

Phone: +86-21-62489820

Fax: $+86-21-62489821$ 
(C) 2008 The Author(s). Licensee IntechOpen. This chapter is distributed under the terms of the Creative Commons Attribution-NonCommercialShareAlike-3.0 License, which permits use, distribution and reproduction for non-commercial purposes, provided the original is properly cited and derivative works building on this content are distributed under the same license. 